\title{
HPV e o câncer de orofaringe
}

- Juliana Milioli Voltolini Residência em Alta Complexidade em Saúde, Hospital Universitário Polydoro Ernani de São Thiago, Florianópolis, SC, Brasil • Felipe Daniel Búrigo dos Santos Residência em Cirurgia e Traumatologia Bucomaxilofacial, Universidade Federal de Santa Catarina, Florianópolis, SC, Brasil

RESUMO | Objetivo: O objetivo deste artigo é revisar a produção científica internacional e nacional sobre o HPV e o câncer de cabeça e pescoço, com foco nos tumores de orofaringe. Materiais e Métodos: Trata-se de uma revisão sistemática de literatura, em que foi realizada uma busca bibliográfica por meio de recursos eletrônicos obtidos, em sua maioria, na base de dados PubMed, publicados entre o período de 1999 a 2019. Resultados: É possível afirmar que o HPV é um fator para a formação câncer de células espinocelulares da orofaringe(CCEO) e, entre os tipos de HPV, o HPV-16 é o tipo mais comum relacionado ao SCCOP. Conclusões: Tendo em vista o alto índice de morbidade e a alta taxa de pacientes que possuem o diagnóstico de câncer de cabeça e pescoço tardio, deve-se levar em consideração que o diagnóstico precoce é de suma importância para estes pacientes.

DESCRITORES | Neoplasias Orofaríngeas; HPV; Oncologia Médica.

ABSTRACT | Hpv and oropharyngeal cancer - Objectives: To review the international and national scientific production on Human Papillomavirus (HPV) and head and neck cancer, focusing on oropharyngeal tumors. Materials and Method: This is a systematic literature review conducted in the PubMed database for electronic resources published between 1999 and 2019. Results: The results indicate that HPV is a factor for the formation of oropharyngeal squamous cells carcinoma (OPSCC), being HPV-16 the most common type of HPV related to OPSCC. Conclusions: Before the elevated rates of morbidity and late diagnosis of head and neck cancer, early diagnosis plays a key role in patients affected with this condition.

DESCRITORES | Oropharyngeal Neoplasms; HPV; Medical Oncology

AUTOR CORRESPONDENTE | Juliana Milioli Voltolini Universidade Federal de Santa Catarina (UFSC) - Rua Dr. Jose de Prata, 330 Criciúma, SC, Brasil • $\mathbf{8 8 8 0 2 2 4}$ E-mail: julimilioli@hotmail.com

- Recebido 18 abril, 2021 • Aceito 30 junho, 2021

- Dol http://dx.doi.org/10.11606/issn.2357-8041.clrd.2019.184527 


\section{INTRODUÇÃO}

O tumor de cabeça e pescoço é a sexta neoplasia maligna mais comum em todo o mundo, com uma ocorrência anual em torno de 600.000 e com mais de 300.000 óbitos por ano. ${ }^{1}$ No Brasil, estes tumores estão entre os dez tipos mais predominantes de câncer. ${ }^{2}$ Um dos mais relevantes problemas de saúde pública é o carcinoma espinocelular de cabeça e pescoço (HNSCC), o tipo histológico mais frequente, representando 90\% dos casos. Estão envolvidos nesta classe de tumor de cabeça e pescoço regiões anatômicas como cavidade oral, orofaringe, hipofaringe, nasofaringe e laringe. ${ }^{3,4}$

Os principais fatores pré-oncogênicos relacionados ao desenvolvimento do HNSCC são o tabaco e o álcool, que podem agir em sinergia, aumentando seus efeitos. Especialmente, para os carcinomas de orofaringe, o vírus do papiloma humano (HPV) também tem grande influência ${ }^{4,5}$. Nas últimas três décadas, a incidência de câncer de cabeça e pescoço, em geral, está diminuindo, devido em grande parte ao maior controle do tabagismo na população, ${ }^{6,7}$ enquanto a incidência de câncer de orofaringe vem aumentando. ${ }^{8}$

No Brasil, o número de casos totais de câncer até 2018 era de 559,371, com prevalência de mortalidade de $43,56 \%$. Atualmente as neoplasias em orofaringe afetam de 11 a 13 pessoas a cada 100.000 habitantes, com, em média, aproximadamente 11 a 15 mil novos casos por ano. ${ }^{2}$

O câncer de orofaringe e de colo do útero são os dois tipos mais comuns relacionados ao HPV, representando cerca de $71 \%$ de todos os tumores associados ao papiloma vírus nos EUA. ${ }^{9}$ Pacientes que apresentam câncer orofaríngeo associados ao diagnóstico de HPV positivo apresentam um melhor prognóstico quando comparados ao câncer orofaríngeo isolado. ${ }^{10}$

A partir dessa perspectiva, este estudo tem como objetivo analisar a literatura específica sobre o assunto e elucidar o potencial do papiloma vírus humano na formação do câncer, em específico, o de orofaringe.

\section{DISCUSSÃO}

O papiloma vírus humanos (HPV) pode causar infecções responsáveis por diferentes manifestações clínicas, desde verrugas até um tumor invasivo. Uma dúzia de tipos de HPV de alto risco são poderosos carcinógenos humanos. ${ }^{11-13}$

No trato aerodigestivo superior, o HPV-16 é reconhecido como a causa de uma proporção crescente de câncer, como o de orofaringe. ${ }^{14}$

O papiloma vírus humanos é um vírus epiteliotrópico de DNA de cadeia dupla com mais de 200 genótipos já identificados. ${ }^{4,15,16} \mathrm{O}$ vírus pode se apresentar como de baixo risco, não oncogênico, que são responsáveis por lesões benignas, e genótipos de HPV de alto risco, oncogênicos, relacionado a alguns tipos de tumores malignos. ${ }^{3,4,16,17}$

A exposição a este vírus é muito comum, podendo ela acontecer pelo contato orogenital ou por outro comportamento sexual, sendo ele reconhecido como uma infecção sexualmente transmissível (IST). ${ }^{18,19}$

O câncer de orofaringe (OPC) induzido por HPV abrange tanto homens como mulheres, porém sua maior incidência ocorre em homens. ${ }^{20,21}$ Isto pode ser explicado pela maior transmissão do HPV pela relação sexual vaginal-oral em vez da peniana oral. Por conta disso, segundo a literatura, o risco de OPC é maior em mulheres homossexuais do que em mulheres heterossexuais. Este resultado não indica o mesmo para relações em que homens possuem relações com outros homens. ${ }^{17,22}$

$\mathrm{O}$ risco diminuído de OPC gerado por HPV em mulheres também pode ser explicado, de forma parcial, pela imunidade adquirida aumentada destas, devido à exposição mais frequente ao HPV na mucosa genital. ${ }^{20}$

A infecção pelo HPV é eliminada pelo sistema imune, entretanto, o contato com um subtipo de alto risco (oncogênico) deste vírus pode gerar uma infecção persistente que futuramente faça surgir neoplasias malignas.,3,23 O HPV está relacionado atualmente como a maior causa de carcinomas em 
jovens que não são fumantes ou que não fazem uso frequente de álcool. ${ }^{24}$

Evidências moleculares proporcionam sustentação para o papel da infecção por HPV oncogênico na carcinogênese de neoplasias de cabeça e pescoço pela inativação das proteínas supressoras tumorais p53 e retinoblastoma. ${ }^{18,25}$ Em decorrência disto, tem-se a desregulação do ciclo celular. ${ }^{18,26}$ Genótipos de HPV oncogênico podem gerar um tumor maligno, porém o HPV-16 se mostra como o mais prevalente, independentemente do local em que o câncer está situado. Mais de 70\% dos cerca de 12.00o tumores provenientes da orofaringe nos Estados Unidos têm como causa o HPV, com 90\% destes causados pelo HPV-16 e o os outros, por outros tipos de HPV de alto risco. ${ }^{15,25-27}$

São características dos pacientes com câncer de células espinocelulares da orofaringe (SCCOP) a agressividade do tumor local, a alta frequência de segundo tumor primário, uma taxa moderada de recorrência e uma alta frequência de comorbidades médicas. ${ }^{28}$ Para o tratamento de tal patologia podem ser usados, em combinação ou individualmente, cirurgia, radioterapia e quimioterapia. A recorrência segue como um grande problema clinicamente para a mortalidade. O HPV vem sendo apontado como um fator de risco etiológico para SCCOP, de modo que cerca de 50-80\% dos pacientes com SCCOP são HPV positivo [HPV (+)].29,30 Entre os tipos de HPV, o HPV-16 é o tipo mais comum de HPV de alto risco no SCCOP, representando aproximadamente 90-95\% dos HPV (+) SCCOP..$^{30,31}$

Rietbergen et al. ${ }^{32}$ relataram em seu artigo que 281 de 926 (30,3\%) SCCOP foram positivos para HPV. Além disso, foi observado um aumento na prevalência de SCCOP positivos para HPV de 14,0\%, em 2000, para 48,1\%, em 2015.

$\mathrm{O}$ aumento do câncer de células espinocelulares da orofaringe positivo para HPV, bem como seu aumento geral, corresponde a dados publicados nos Estados Unidos e em outros países europeus. Sobre a prevalência global de HPV no carcinoma de células espinocelulares da cavidade oral (OSCC), foi feita uma revisão e uma meta-análise na qual revelou-se que a prevalência foi de $24,2 \%$ em média, comparado com $45,8 \%$ na orofaringe. ${ }^{33-35}$

Alguns sintomas, que muitas vezes podem aparecer associados, podem estar relacionados ao aparecimento de um tumor em região de cabeça e pescoço, como: emagrecimento, dor, lesão, disfagia, disfonia, rouquidão, otalgia e hemorragia oral. Segundo Figueiredo, ${ }^{36}$ estes sintomas se apresentam de forma mais comum em pacientes em que o tumor acometeu sítios anatômicos como orofaringe, hipofaringe, língua e laringe. Tais sintomas são relacionados ao avanço da patologia e, de mesmo modo, ao atraso na procura de um tratamento adequado para esta neoplasia.

Na maioria dos casos, os pacientes com tumor de orofaringe são diagnosticados tardiamente. Petito et al., ${ }^{37} \mathrm{em}$ seu estudo, demonstrou que dos tumores positivos para HPV, 4,8\% se encontravam nos estágios I/II e 95,2\% estavam nos estágios III/IV. ${ }^{37}$

Mesmo tendo os tumores de orofaringe diversas entidades clínicas quando comparados com tumores de outras localidades e relacionados com o HPV, possuem todos o mesmo tipo de tratamento. Visto que há melhor resposta ao tratamento e melhor prognóstico do paciente com tumor HPV positivo nos dias de hoje, estuda-se uma diminuição na intensidade do tratamento antineoplásico com a finalidade de reduzir a toxicidade do tratamento em períodos longos e para avaliar uma resposta individual da terapia. ${ }^{38}$

As terapias comumente usadas são a cirurgia, em conjunto com a radioterapia e a quimioterapia, e a imunoterapia como adjuvante. Porém, estas formas de tratamento se encontram longe de produzir resultados considerados satisfatórios. Tumores na região da orofaringe e da cavidade oral apresentam rápida ação de metástase, implicando muita das vezes em tratamentos cirúrgicos agressivos. ${ }^{39-41}$ 
Deve-se também ser levado em consideração que, em geral, estas neoplasias são diagnosticadas tardiamente, o que impede o uso de abordagens mais conservadoras. ${ }^{5,42}$

As formas de tratamento utilizadas interferem na maioria das vezes de forma provisória ou definitiva em atividades cotidianas como fonação, mastigação e deglutição, o que gera comorbidades iatrogênicas potencialmente importantes nestes pacientes. ${ }^{43}$ Estas disfunções, devido ao tratamento antineoplásico, podem fazer com que frequentemente estes pacientes tenham a necessidade do uso de opções como a gastrostomia percutânea. ${ }^{44,45}$

Nestes pacientes também há alterações importantes na fisionomia e na fala, assim como ocasionar dor, dispneia, fadiga, ansiedade, depressão e isolamento social e outras sintomatologias, como a mucosite oral, hipossialia/xerostomia, disgeusia e infecções oportunistas proveniente do tratamento antineoplásico. ${ }^{46-48}$

O tratamento oncológico pode produzir grande impacto na vida dos pacientes e na sua qualidade de vida, tanto durante o tratamento quando posterior a ele.

A taxa de sobrevida em pacientes afetados pelo tumor de cabeça e pescoço em relação a tumores secundários relacionados com o papiloma vírus ainda é desconhecida nos dias de hoje. Foi relatado em um estudo que tumores secundários na parte contralateral das tonsilas passam a ocorrer em $4 \%$ (6/135) em pacientes acometidos por neoplasia de células escamosas tonsilares associados ao HPV. 49

O prognóstico destes tumores se relaciona de forma íntima com o estadiamento e com o grupo de risco atribuído a ele, destacando-se o constante retardo no diagnóstico inicial..$^{5}$.

Em geral, a sobrevida destes pacientes é considerada superior à de pacientes com neoplasias HPV-negativos, que têm como uma das características principais a baixa sobrevida. ${ }^{11} \mathrm{~A}$ American Cancer Association (ACA) afirma que a média de sobrevida aos 5 e 10 anos para neoplasias das regiões anatômicas da cavidade oral e de orofaringe é $63 \%$ e $52 \%$, respectivamente. ${ }^{52}$

\section{CONCLUSÃO}

Tendo em vista o alto índice de morbidade e a alta taxa de pacientes que possuem um diagnóstico tardio, deve-se levar em consideração que o diagnóstico precoce é de suma importância para pacientes deste tipo de câncer, podendo muitas vezes definir a sobrevida e a forma de tratamento deste. Profissionais como o cirurgião-dentista, que possuem íntimo contato com a região de cabeça e do pescoço, devem estar atentos. É necessário fazer exame clínico e anamnese minuciosa e avisar ao paciente dos fatores pré-oncogênicos falados por ele na anamnese.

Os fatores pré-oncogênicos estão presentes no cotidiano, apresentando-se de variadas formas. Sendo assim, faz-se necessário cada vez mais estudos para que se possa estabelecer um perfil de pacientes mais susceptíveis e compreender melhor a influência dos fatores químicos, físicos e biológicos que envolvem a formação de neoplasias de cabeça e pescoço, tal como a melhor forma de tratamento para as especificidades de cada patologia e a particularidades de cada sítio anatômico.

\section{REFERÊNCIAS}

1. Lewis A, Kang R, Levine A, Maghami E. The New Face of Head and Neck Cancer: The HPV Epidemic. Oncology (Williston Park). 2015;29(9):616-26.

2. Instituto Nacional do Câncer (BR). Homepage [Internet]. Rio de Janeiro: INCA; 2009 [citado em 15 maio 2018]. Disponível em: www.inca.gov.br

3. Albano PM, Holzinger D, Salvador C, Orosa J 3rd, Racelis S, Leaño M, Sanches D Jr, et al. Low prevalence of human papilloma virus in head and neck squamous cell carcinoma in the north west region of the Philippines. PLoSOne. 2017;12(2):e0172240. doi: 10.1371/journal.pone.0172240.

4. Mehanna H, Paleri V, West C, Nutting C. Head and neck cancer - Part 1: Epidemiology, presentation, and prevention. BMJ. 2010;341:c4684. doi: 10.1136/bmj.c4684. 
5. Warnakulasuriya S. Global epidemiology of oral and oropharyngeal cancer. Oral Oncol. 2009;45(4-5):309-16. doi: 10.1016/j.oraloncology.2008.06.002.

6. Siegel R, Naishadham D, Jemal A. Cancer Statistics, 2012. CA Cancer J Clin. 2012;62(1):10-29. doi: 10.3322/caac.20138t.

7. Jemal A, Bray F, Center MM, Ferlay J, Ward E, Forman D. Global cancer statistics. CA Cancer J Clin. 2011;61(2):69-90. doi: 10.3322/caac.20107.

8. Chaturvedi AK, Engels EA, Anderson WF, Gillison ML. Incidence trends for human papillomavirus-related and -unrelated oral squamous cell carcinomas in the United States. J Clin Oncol. 2008;26(4):612-9. doi: 10.1200/JCO.2007.14.1713.

9. Viens LJ, Henley SJ, Watson M, Markowitz LE, Thomas CC, Thompson TD, et al. Human papilomavirus: associated cancers: United States, 2008-2012. MMWR Morb Mortal Wkly Rep. 2016;65(26):661-6. doi: 10.15585/mmwr.mm6526a1.

10. Wirth L, Burtness B, Nathan CA, Grégoire V, Richmon J. Point/ Counterpoint: Do We De-escalate Treatment of HPV-Associated Oropharynx Cancer Now? And How? Am Soc Clin Oncol Educ Book. 2019;39:364-72. doi: 10.1200/EDBK_238315.

11. IARC Working Group on the Evaluation of Carcinogenic Risks to Humans. Biological agents: Volume 10oB: A review of human carcinogens. IARC Monogr Eval Carcinog Risks Hum. 2012;100(Pt B):1-441.

12. Bedell SL, Goldstein LS, Goldstein AR, Goldstein AT. Cervical Cancer Screening: past, present, and future. Sex Med Rev. 2020;8(1):28-37 doi: 10.1016/j.sxmr.2019.09.005.

13. Walboomers JM, Jacobs MV, Manos MM, Bosch FX, Kummer JA, Shah KV, et al. Human papillomavirus is a necessary cause of invasive cervical cancer worldwide. J Pathol. 1999;189(1):12-9. doi: 10.1002/(SICI)10969896(199909)189:1<12::AID-PATH431>3.0.CO;2-F.

14. Martel C, Plummer M, Vignat J, Franceschi S. Worldwide burden of cancer attributable to HPV by site, country and HPV type. Int J Cancer. 2017;141(4):664-70. doi: 10.1002/ijc.30716.

15. Berman TA, Schiller JT. Human papillomavirus in cervical cancer and oropharyngeal cancer: One cause, two diseases. Cancer. 2017;123(12):2219-29. doi: 10.1002/cncr.30588.

16. Ferlay J, Soerjomataram I, Ervik M, Dikshit R, Matthers C, Rebelo M, et al. GLOBOCAN 2012: Estimated Cancer Incidence, Mortality and Prevalence Worldwide in 2012 v1.o. Lyon: IARC; 2013.

17. D’Souza G, Cullen K, Bowie J, Thorpe R, Fakhry C. Differences in oral sexual behaviors by gender, age, and race explain observed differences in prevalence of oral human papillomavirus infection. PLoSOne. 2014;9(1):19-21. doi: 10.1371/ journal.pone.0086023.

18. Gao G, Smith DI. Human Papillomavirus and the Development of Different Cancers. Cytogenet Genome Res. 2016; 150(3-4):185-93. doi: 10.1159/000458166.
19. Taberna M, Inglehart RC, Pickard RK, Fakhry C, Agrawal A, Kats ML, et al. Significant changes in sexual behavior after a diagnosis of human papillomavirus-positive and human papillomavirus-negative oral cancer. Cancer. 2017;123(7):115665. doi: 10.1002/cncr.30564.

20. Combes JD, Chen AA, Franceschi S. Prevalence of human papillomavirus in cancer of the oropharynx by gender. Cancer Epidemiol Biomarkers Prev. 2014;23(12):2954-8. doi: 10.1158/1055-9965.EPI-14-0580.

21 Li H, Torabi SJ, Yarbrough WG, Mehra S, Osborn HA, Judson B. Association of Human Papillomavirus Status at Head and Neck Carcinoma Subsites With Overall Survival. JAMA Otolaryngol Head Neck Surg. 2018;144(6):519-525. doi: 10.1001/ jamaoto.2018.0395.

22. Saunders CL, Meads C, Abel GA, Lyratzopoulos G. Associations Between Sexual Orientation and Overall and SiteSpecific Diagnosis of Cancer: Evidence From Two National Patient Surveys in England. J Clin Oncol. 2017;35(32):365461. doi: 10.1200/JCO.2017.72.5465.

23. Gillison ML, Castellsagué X, Chaturvedi A, Goodman MT, Snijders P, Tommasino M, et al. Eurogin Roadmap: comparative epidemiology of HPV infection and associated cancers of the head and neck and cervix. Int J Cancer. 2014;134(3):497507. doi: 10.1002/ijc.28201.

24. Organización Panamericana de la Salud. Classificación estadística internacional de enfermedades y problemas relacionados con la salud: décima revisión. Washington, DC: OPS; 1995 .

25. Agalliu I, Gapstur S, Chen Z, Wang T, Anderson RL, Teras $\mathrm{L}$, et al. Associations of Oral $\alpha-, \beta$-, and $\gamma$-Human papillomavirus types with risk of incident head and neck cancer. JAMA Oncol. 2016;2(5):599-606. doi: 10.1001/jamaoncol.2015.5504.

26. Benson E, Li R, Eisele D, Fakhry C. The clinical impact of HPV tumor status upon head and neck squamous cell carcinomas. Oral Oncol. 2014;50(6):565-74. doi: 10.1016/j.oraloncology.2013.09.008.

27. Fakhry C, Westra WH, Wang SJ, van Zante A, Zhang Y, Rettig $\mathrm{E}$, et al. The prognostic role of sex, race, and human papillomavirus in oropharyngeal and nonoropharyngeal head and neck squamous cell cancer. Cancer. 2017;123(9):1566-75. doi: 10.1002/cncr.30353.

28. Reuschenbach M, Wagner S, Würdemann N, Sharma SJ, Prigge ES, Sauer M, et al. Human papillomavirus and squamous cell cancer of the head and neck region: Prognostic, therapeutic and prophylactic implications. HNO. 2016;64(7):450-9. doi: 10.1007/s00106-016-0123-0.

29. Sturgis EM, Cinciripini PM. Trends in head and neck cancer incidence in relation to smoking prevalence: an emerging epidemic of human papillomavirus-associated cancers? Cancer. 2007;110(7):1429-35. doi: 10.1002/cncr.22963. 
30. Marur S, D'Souza G, Westra WH, Forastiere AA. HPV-associated head and neck cancer: a virus-related cancer epidemic. Lancet Oncol. 2010;11(8):781-9. doi: 10.1016/S14702045(10)70017-6.

31. Hammarstedt L, Lindquist D, Dahlstrand H, Romanitan M, Dahlgren LO, Joneberg J, et al. Human papillomavirus as a risk factor for the increase in incidence of tonsillar cancer. Int J Cancer. 2006;119(11):2620-3. doi: 10.1002/ijc.22177.

32. Rietbergen MM, van Bokhoven AA, Lissenberg-Witte BI, Heideman DAM, Leemans CR, Brakenhoff RH, et al. Epidemiologic associations of HPV-positive oropharyngeal cancer and (pre)cancerous cervical lesions. I Jornal Cancer. 2018;143(2):283-8. doi: 10.1002/ijc.31315.

33. Ang KK, Harris J, Wheeler R, Weber R, Rosenthal DI, NguyenTân PF, et al. Human papillomavirus and survival of patients with oropharyngeal cancer. N Engl J Med. 2010;363(1):24-35. doi: 10.1056/NEJMoao912217.

34. Ndiaye C, Mena M, Alemany L, Arbyn M, Castellsagué X, Laporte L, et al. HPV DNA, E6/E7 mRNA, and p16(INK4a) detection in head and neck cancers: a systematic review and meta-analysis. Lancet Oncol. 2014;15(12):1319-31. doi: 10.1016/S1470-2045(14)70471-1.

35. Klussmann JP, Preuss SF, Speel EJ. Human papillomavirus and cancer of the oropharynx. Molecular interaction and clinical implications. HNO. 2009;57(2):113-22. doi: 10.1007/ soo106-008-1867-y.

36. Figueiredo AM. Prevalências e características clínico-epidemiológicas do câncer bucal e de cabeça e pescoço no Hospital Estadual de Bauru/SP [tese]. São Paulo: Universidade de São Paulo; 2013.

37. Petito G, Carneiro MAS, Santos SHR, Silva AMTC, Alencar RC, Gontijo AP, et al. Human papillomavirus in oral cavity and oropharynx carcinomas in the central region of Brazil. Braz J Otorhinolaryngol. 2017;83(1):38-44. doi: 10.1016/j. bjorl.2016.01.004.

38. Caley A, Evans M, Powell N, Paleri V, Tomkinson A, Urbano TG, et al. Multicentric human papillomavirus-associated head and neck squamous cell carcinoma. Head Neck. 2015;37(2):202-8. doi: 10.1002/hed.23584.

39. Cardoso MFA, Novikoff S, Tresso A, Segreto RA, Cervantes $\mathrm{O}$. Prevenção e controle das sequelas bucais em pacientes irradiados por tumores de cabeça e pescoço. Radiol Bras. 2005;38(2):107-15. doi: 10.1590/So10o39842005000200006.

40. Graeff A, de Leeuw JR, Ros WJ, Hordijk GJ, Blijham GH, Winnubst JA. Pretreatment factors predicting quality of life after treatment for head and neck cancer. Head Neck. 2000;22(4):398407. doi: 10.1002/1097-0347(200007)22:4<398::aidhed14>3.0.co;2-v.
41. Chinn SB, Myers JN. Oral cavity carcinoma: current management, controversies, and future directions. J Clin Oncol. 2015;33(29):3269-76. doi: 10.1200/JCO.2015.61.2929.

42. Burd EM, Dean CL. Human papillomavirus. Microbiol Spectr. 2016;4(4). doi: 10.1128/microbiolspec.DMIH2-0oo1-2015.

43. Yeh DH, Tam S, Fung K, MacNeil SD, Yoo J, Winquist E, et al. Transoral robotic surgery vs. radiotherapy for management of oropharyngeal squamous cell carcinoma: a systematic review of the literature. Eur J Surg Oncol. 2015;41(12):1603-14. doi: 10.1016/j.ejso.2015.09.007.

44. Heijnen BJ, Speyer R, Kertscher B, Cordier R, Koetsenruijter KWJ, Swan K, et al. Dysphagia, Speech, Voice, and Trismus following Radiotherapy and/or Chemotherapy in Patients with Head and Neck Carcinoma: Review of the Literature. Biomed Res Int. 2016;2016:6086894. doi: 10.1155/2016/6086894.

45. King SN, Dunlap NE, Tennant PA, Pitts T. Pathophysiology of Radiation-Induced Dysphagia in Head and Neck Cancer. Dysphagia. 2016;31(3):339-51. doi: 10.1007/s00455-016-9710-1.

46. Connor NP, Cohen SB, Kammer RE, Sullivan PA, Brewer KA, Hong TS, et al. Impact of conventional radiotherapy on health-related quality of life and critical functions of the head and neck. Int J Radiat Oncol Biol Phys. 2006;65(4):1051-62. doi: 10.1016/j.ijrobp.2006.01.054.

47. Knox JJ, Puodziunas AL, Feld R. Chemotherary-induced oral mucositis: Prevention and management. Drugs Aging. 2000;17(4):257-67. doi: 10.2165/oooo2512-200017040-00002.

48. Stoeckli SJ, Guidicelli M, Schneider A, Huber A, Schmid S. Quality of life after treatment for early laryngeal carcinoma. Eur Arch Otorhinolaryngol. 2001;258(2):96-9. doi: 10.1007/ s004050000307.

49. Joseph AW, Ogawa T, Bishop JA, Lyford-Pike S, Chang X, Phelps TH, et al. Molecular etiology of second primary tumors in contralateral tonsils of human papillomavirus-associated index tonsillar carcinomas. Oral Oncol. 2013;49(3):244-8. doi: 10.1016/j.oraloncology.2012.09.009.

50. Naghavi AO, Echevarria MI, Strom TJ, Abuodeh YA, Ahmed KA, Venkat PS, et al. Treatment delays, race, and outcomes in head and neck cancer. Cancer Epidemiol. 2016;45:18-25. doi: 10.1016/j.canep.2016.09.005

51. Okami K. Clinical features and treatment strategy for HPV-related oropharyngeal cancer. Int $\mathrm{J}$ Clin Oncol. 2016;21(5):827-35. doi: 10.1007/s10147-016-1009-6.

52. American Cancer Society. Cancer Facts \& Figures 2016. Atlanta: American Cancer Society; 2016. 